\title{
Cardiac disease in systemic lupus erythematosus: prospective study of 70 patients
} Ricard Cervera, Josep Font, Carles Paré, Manuel Azqueta, Félix Pérez-Villa,
Alfons López-Soto, Miguel Ingelmo

\begin{abstract}
A prospective two dimensional and Doppler echocardiographic study of 70 consecutive patients with systemic lupus erythematosus (SLE) and 40 controls was carried out. Forty patients $(57 \%)$ were found to have echocardiographic disturbances. Valvular abnormalities were detected in 31 patients (44\%) and in only two controls (5\%). Mitral valve abnormalities were the most common findings $(23 / 70(33 \%))$ with mild or moderate regurgitation the most frequent lesion (16\% and $9 \%$ respectively). Three patients (4\%) had a morphological echocardiographic pattern suggestive of noninfective verrucous vegetations affecting the mitral valve. No patient had haemodynamically significant clinical valve disease. Pericardial effusion was identified in 19 patients $(27 \%)$, of whom 14 had mild and clinically silent disease. Myocardial abnormalities were found in 14 patients $(20 \%)$, but clinical features of myocardial dysfunction were present in only one. Patients with antiphospholipid antibodies were found to have an increased prevalence of endocardial lesions, mainly valvular regurgitation. It is concluded that the inclusion of echocardiography in a study protocol of patients with SLE can identify an important subset of patients with cardiac abnormalities, many of which are clinically silent. In addition, the association of antiphospholipid antibodies with endocardial lesions suggests that these antibodies may have a prominent role in the pathogenetic mechanisms of heart valve disease in SLE.
\end{abstract}

Systemic Autoimmune Diseases Research Group, Department of Internal Medicine,

Hospital Clínic i

Provincial, Barcelona,

Spain

R Cervera

J Font

A López-Soto

$M$ Ingelmo

Department of

Cardiology, Hospital

Clínic i Provincial,

Barcelona, Spain

C Paré

M Azqueta

F Pérez-Villa

Correspondence to:

Dr Josep Font,

Department of

Internal Medicine-Unit 1

Hospital Clínic

Provincial, Villarroel 170

08036 Barcelona, Spain.

Accepted for publication

26 February 1991
Systemic lupus erythematosus (SLE) is an autoimmune disorder resulting in multisystemic inflammatory damage. Because of the pleomorphic nature of this disease its cardiac manifestations have not always been emphasised. In recent years, however, with prolonged survival and improvement in diagnostic techniques, the cardiac disease associated with SLE has become more apparent. ${ }^{12}$

The most characteristic cardiac abnormalities in SLE are non-infective vegetations (LibmanSacks endocarditis). Although they are present in up to $50 \%$ of patients at necropsy, ${ }^{3}{ }^{4}$ reports of their presence before death are uncommon. In addition, pericarditis is a very common clinical manifestation in SLE, occurring in 20 to $30 \%$ of all patients. ${ }^{5}$ Conversely, myocardial lesions have been rarely documented. Nevertheless, recent introduction of two dimensional and Doppler echocardiography has disclosed a higher prevalence of patients with cardiac abnormalities than previously reported-many of them clinically silent and without manifestations of SLE activity. ${ }^{6-10}$

In the past few years reports have linked the presence of antiphospholipid antibodies-that is, anticardiolipin antibodies and the lupus anticoagulant, with cardiac lesions, such as noninfective vegetations and valvular dysfunctions. ${ }^{11-13}$ Nevertheless, prospective studies of large numbers of unselected patients are few and no definite conclusions have been reached.

Therefore, our study aimed at describing the prevalence of cardiac lesions in a large living group of unselected patients with SLE by means of two dimensional and Doppler echocardiography, and analysing their relation with clinical features of cardiopathy, lupus activity, and the presence of antiphospholipid antibodies.

\section{Patients and methods}

PATIENTS AND CONTROLS

Seventy consecutive white patients with SLE (64 women, six men) attending the Hospital Clínic of Barcelona as inpatients or outpatients, between 1986 and 1989, were studied prospectively. The average age of the patients was 38 (SD 15) years (range 8-73). All patients were questioned by a qualified cardiologist about a history of previous rheumatic fever, infective endocarditis, hypertension, pleuritic or anginal chest pain, and dyspnoea. A conventional cardiovascular physical examination was made and an electrocardiogram and chest radiographs obtained for all patients. No patient had a history suggestive of rheumatic fever or infective endocarditis, and none was specifically referred because of suspected valvular abnormalities. In those with valve thickening and nodular vegetations, infective endocarditis was reasonably ruled out by six negative blood cultures.

In addition, 40 healthy subjects (36 women, four men), matched to the patient group for age and sex (mean age 37 (11) years) served as controls. All were volunteers with no clinical evidence of any cardiac or systemic complaints. Informed consent was obtained from all patients and controls.

DIAGNOSIS AND CLINICAL ACTIVITY OF SLE All the patients included in the study fulfilled the 1982 revised criteria of the American Rheumatism Association for the classification of SLE. ${ }^{14}$ Average duration of SLE was 63 (54) months (range 4-254). Individual clinical 
features of SLE were considered by a qualified doctor if they fulfilled the definitions of the American Rheumatism Association glossary. ${ }^{15}$

To assess disease activity all records were evaluated without knowledge of the echocardiographic findings. Disease was judged to be clinically active if the following signs or symptoms were present: typical dermatitis; arthritis; serositis; central nervous system abnormalities (recent onset of chorea, seizures, psychosis, organic brain syndrome in the absence of offending drugs or known metabolic derangements, embolic cerebral vascular accidents); thrombocytopenia $\left(<100 \times 10^{9}\right.$ platelets/l); haemolytic anaemia; vasculitis (biopsy); or nephritis (recent onset of haematuria ( $>10$ red blood cells/high power field), or casts, or proteinuria $(>500 \mathrm{mg} / 24 \mathrm{~h})$, or a $25 \%$ increase in serum creatinine).

\section{ECHOCARDIOGRAMS}

Two dimensional and Doppler echocardiograms were obtained with a commercially available phased array ultrasonic sector scanner with a $2.5 \mathrm{MHz}$ transducer. Images were analysed according to the recommendations of the American Society of Echocardiography. ${ }^{16}$ Two echocardiologists independently assessed all studies without knowledge of the patients' status. In the event of a disagreement, the two echocardiologists reached a consensus by a joint review of the study.

\section{LABORATORY STUDIES}

Antinuclear antibodies were determined by indirect immunofluorescence using mouse liver as substrate. Antibodies to dsDNA were determined by Farr's ammonium sulphate precipitation technique. Complement components (C3 and $\mathrm{C4}$ ) were estimated by radial immunodiffusion and $\mathrm{CH} 50$ by Lachmann's haemolytic technique. Circulating immune complexes were detected by a microcomplement consumption test. Precipitating antibodies to soluble nuclear and cytoplasmic antigens, including $\operatorname{Ro}(\mathrm{SSA})$, $\mathrm{La}$ (SSB), nRNP, and Sm, were determined by double immunodiffusion (Ouchterlony).

Antibodies to cardiolipin were measured by an enzyme linked immunosorbent assay (ELISA) as described by Loizou et $a l^{17}$ and Gharavi et al ${ }^{18}$ with our minor modifications ${ }^{19}$ and those of Cowchock et al. ${ }^{20}$ The flat bottomed wells of the microtitre plates (Nunc, Denmark) were coated with $30 \mu \mathrm{l} /$ well of cardiolipin (Sigma) suspended in ethanol at a final concentration of $50 \mu \mathrm{g} / \mathrm{ml}$. The test was modified by inclusion of a measurement of binding to the microtitre plates after blocking (with $10 \%$ fetal calf serum in phosphate buffered saline for two hours) without added cardiolipin. Tests were performed in duplicate, both with and without phospholipid. Results were expressed as a binding index (BI) given by the formula:

$$
\mathrm{BI}(\text { specific })=\mathrm{BI}(\text { total })-B I(\text { non-specific })
$$

where BI $($ total)=average value for samples from the wells containing cardiolipin; BI (non- specific)=average value for samples from the wells containing no cardiolipin.

A study of 50 serum samples from normal controls showed a log-normal distribution of either IgG or IgM anticardiolipin antibody titres. Normal values were considered when the logarithms of the specific BI were below the 98th percentile of cumulative normal distribution. The IgG anticardiolipin antibody titre was regarded as positive if a specific BI greater than 2.85 (SD 3.77) was detected. A specific BI greater than 4.07 (3.90) was defined as positive for IgM anticardiolipin antibody. Lupus anticoagulant activity was detected by the method of Exner et al. ${ }^{21}$

\section{STATISTICAL ANALYSIS}

Conventional $\chi^{2}$ and Fisher's exact tests were used for analysing qualitative differences, Student's $t$ test for comparison of means in large samples of similar variance, and the nonparametric Mann-Whitney $U$ test in small samples for comparing age differences between groups. Statistical significance was defined as $\mathrm{p}<0.05$.

\section{Results}

\section{ECHOCARDIOGRAPHIC ABNORMALITIES AND}

CLINICAL FEATURES OF CARDIOPATHY

Forty patients with SLE (57\%) were found to have echocardiographic abnormalities. There was no significant difference in sex, age, and duration of disease between those with and without echocardiographic abnormalities.

Valvular abnormalities were detected in 31 patients $(44 \%)$ and in only two controls $(5 \%)$ $(\mathrm{p}<0.001)$ (table 1). Mitral valve abnormalities were the most common finding $(23 / 70(33 \%))$, with mild or moderate regurgitation the most common lesion (11/70 (16\%) and 6/70 (9\%) respectively). Three patients $(4 \%)$ had a morphological echocardiographic pattern suggestive of non-infective verrucous vegetations affecting the mitral valve. A Doppler pattern of mitral regurgitation was present in all three cases. When patients with mitral valve prolapse

Table 1 Echocardiographic valvular abnormalities in patients with systemic lupus erythematosus $(S L E)$ and controls. Results are shown as number (percentage) of patients

\begin{tabular}{lll}
\hline Abnormalities & $\begin{array}{l}\text { Patients with } \\
S L E(n=70)\end{array}$ & $\begin{array}{l}\text { Controls } \\
(n=40)\end{array}$ \\
\hline Valvular & $31(44)$ & $2(5)$ \\
Mitral valve & $23(33)$ & $2(5)$ \\
Vegetations & $3(4)$ & 0 \\
Regurgitation & $20(29)$ & $2(5)$ \\
$\quad$ Mild & $11(16)$ & $2(5)$ \\
Moderate & $6(9)$ & 0 \\
Severe & $3(4)$ & 0 \\
Stenosis & $2(3)$ & 0 \\
Mild & $2(3)$ & 0 \\
Calcification & $2(3)$ & 0 \\
Thickening without dysfunction & $2(3)$ & 0 \\
Prolapse & $2(3)$ & $2(5)$ \\
Aortic valve & & \\
Regurgitation & $9(13)$ & 0 \\
$\quad$ Mild & $5(7)$ & 0 \\
Stenosis & $5(7)$ & 0 \\
Mild & $1(1)$ & 0 \\
Calcification & $1(1)$ & 0 \\
Thickening without dysfunction & $1(6)$ & 0 \\
Tricuspid valve & $1(1)$ & 0 \\
Regurgitation & $4(6)$ & 0 \\
Mild & $4(6)$ & 0 \\
\hline & $4(6)$ & 0 \\
\hline & &
\end{tabular}


and mitral and aortic valve thickening or calcification without concomitant features of valvular dysfunction were excluded the total number of lupus patients with significant echocardiographic valvular lesions was 21 (prevalence 30\%). However, only those with non-infective vegetations and a further group of five patients with mitral or aortic valve dysfunctions $(11 \%)$ had systolic or diastolic murmurs, or both. No patient had haemodynamically significant clinical valve disease.

Pericardial effusion was present in 19 patients (27\%) but in no controls $(\mathrm{p}<0.001)$ (table 2$)$. Five $(7 \%)$ had moderate or severe and $14(20 \%)$ mild effusion. Only patients with moderate and severe pericardial effusion had clinical and electrocardiographic features of pericarditis.

Myocardial abnormalities were found in 14 patients $(20 \%)$ but in no controls $(p<0.001)$ (table 3). Clinical features of myocardial dysfunction, however, were present in only one patient with an echocardiographic pattern suggestive of myocarditis.

\section{ECHOCARDIOGRAPHIC ABNORMALITIES AND SLE} ACTIVITY

Systemic lupus erythematosus was active in 39 patients $(56 \%)$, of whom $26(67 \%)$ had echocardiographic abnormalities, including pericardial effusion (19 patients), non-infective vegetations (three), mild aortic valve regurgitation (two), moderate mitral valve regurgitation (one), mitral valve thickening without dysfunction (one), and myocarditis (one).

\section{ECHOCARDIOGRAPHIC ABNORMALITIES AND} ANTIPHOSPHOLIPID ANTIBODIES

Antiphospholipid antibodies were detected in 23 patients (33\%). Patients with these antibodies had a significantly increased prevalence of endocardial lesions ( 15 out of 23 positive, $\mathrm{p}<0.05$ ), mainly valvular (mitral or aortic valve) regurgitation (with or without vegetations) (13 out of 23 positive, $p<0.05$ ), compared with patients without antiphospholipid antibodies. No relation between antiphospholipid antibodies and pericardial or myocardial lesions was found.

Table 2 Echocardiographic pericardial abnormalities in patients with systemic lupus erythematosus (SLE) and controls. Results are shown as number (percentage) of patients

\begin{tabular}{lll}
\hline Abnormalities & $\begin{array}{l}\text { Patients with } \\
\text { SLE }(n=70)\end{array}$ & $\begin{array}{l}\text { Controls } \\
(n=40)\end{array}$ \\
\hline Pericardial effusion & $19(27)$ & 0 \\
Mild & $14(20)$ & 0 \\
Moderate & $3(4)$ & 0 \\
Severe & $2(3)$ & 0 \\
\hline
\end{tabular}

Table 3 Echocardiographic myocardial abnormalities in patients with systemic lupus erythematosus (SLE) and controls. Results are shown as number (percentage) of patients

\begin{tabular}{lll}
\hline Abnormalities & Patients with & $\begin{array}{l}\text { Controls } \\
(n=40)\end{array}$ \\
\hline Myocardial & $1(n=70)$ & \\
Left ventricle enlargement & $14(20)$ & 0 \\
Left ventricle hypertrophy & $6(10)$ & 0 \\
Myocarditis & $6(9)$ & 0 \\
\hline
\end{tabular}

Discussion

In this echocardiographic study we found a high prevalence of cardiac disturbances (40/70 $(57 \%))$. Valvular abnormalities, mainly mitral and aortic dysfunctions, were the most common finding $(21 / 70(44 \%))$. No patient had haemodynamically significant clinical valve disease. In addition, pericardial effusion was a common echocardiographic finding (19/70 (27\%)), but significant pericardial disease was found in only $5 / 70(7 \%)$ patients. Conversely, significant myocardial abnormalities were less common $(14 / 70(20 \%))$, and only one patient with an echocardiographic pattern of myocarditis developed clinical features of myocardial dysfunction. Interestingly, most patients with SLE activity had echocardiographic abnormalities, raising the importance of cardiac disease in the spectrum of SLE manifestations.

Study of heart valve lesions in SLE is of paramount importance. Whereas mitral valve prolapse is commonly found in a normal population as well as in patients with SLE, other valvular dysfunctions, such as non-infective vegetations and mitral or aortic dysfunctions, are most commonly found in patients with this disorder, ${ }^{6-10}$ as we found in this study. Noninfective vegetations, first described by Libman and Sacks in $1924,{ }^{22}$ are the most characteristic valvular lesions of SLE. Microscopically, they consist of proliferating and degenerating cells, fibrin, fibrous tissue, and occasional haematoxylin bodies. Their most common locations are at the valve margins and on chordae tendineae, papillary muscles, and mural endocardium. $^{12}$ Early reports suggested a predominance of tricuspid disease, but more recent series indicate a higher prevalence of mitral valve vegetations, ${ }^{6-10}$ as found in our patients. Although non-infective vegetations in SLE rarely result in haemodynamically significant valve disease, there are a number of reported cases describing the occurrence of valvular stenosis and regurgitation as a result of Libman-Sacks endocarditis. ${ }^{23-25}$ In fact, most of the valvular dysfunctions in young lupus patients without a history of rheumatic fever or infective endocarditis are probably due to previous silent Libman-Sacks endocarditis. Thromboembolism is a rare consequence of non-infective endocarditis, but pulmonary, cerebral, and coronary embolisation of tissue from these sterile vegetations has been documented. ${ }^{26}$ In addition, Libman-Sacks endocarditis probably predisposes to infective endocarditis. ${ }^{27}$

The association of antiphospholipid antibodies with venous or arterial thrombosis, or both, recurrent fetal loss, and thrombocytopenia has been well documented in several studies. ${ }^{20}$ 28-30 It is postulated that antiphospholipid antibodies may cause these disorders either through platelet activation by binding to platelet phospholipids $^{31}$ or through inhibition of prostacyclin production in the endothelium. ${ }^{32}$ Furthermore, there have been several reports of the possible association of antiphospholipid antibodies with endocardial lesions, such as non-infective vegetations and valvular dysfunctions. ${ }^{11-13}$ In this study we confirmed the 
association of antiphospholipid antibodies with acquired endocardial disease, including mitral or aortic valve regurgitation, probably due to previous silent non-infective endocarditis.

The aetiopathogenesis of these valvular lesions in SLE and their relation with antiphospholipid antibodies is as yet unknown. Nevertheless, Shapiro et al found selective deposition of immunoglobulins and complement along the vessel walls of vegetations, ${ }^{33}$ suggesting that circulating immune complexes may participate in the growth and proliferation of these noninfective vegetations. The pathogenetic role of antiphospholipid antibodies remains to be elucidated, but our results give food for thought about the possibility that antiphospholipid antibodies may contribute to the pathogenetic mechanisms of these valvular lesions by promoting thrombus formation.

In conclusion, the inclusion of two dimensional and Doppler echocardiography in a study protocol of patients with SLE can identify an important subset of patients with cardiac abnormalities. Clinically significant lesions are uncommon, however, usually occurring in patients with SLE activity. In addition, the finding of an association between antiphospholipid antibodies and endocardial lesions suggests the possibility of a prominent role for these antibodies in the pathogenetic mechanisms of heart valve disease in lupus.

Supported by grants DGCYT PM 89-0108 and FISS 92-0385.

1 Doherty N E, Siegel R J. Cardiovascular manifestations of systemic lupus erythematosus. Am Heart 7 1985; 110 systemic

2 Ansari A, Larson P H, Bates H D. Cardiovascular manifestations of systemic lupus erythematosus. Prog Cardiovas Dis 1985; 27: 421-34.

3 Bulkley B H, Roberts W C. The heart in systemic lupus erythematosus and the changes induced in it by corticosteroid therapy. A study of 36 necropsy patients. Am $\mathcal{J} M e d$ 1975; 58: 243-64

4 Kong T Q, Kellum R E, Haserick J R. Clinical diagnosis of cardiac involvement in systemic lupus erythematosus: a correlation of clinical and autospy findings in thirty patients. Circulation 1962; 26: 7-11.

patients. Circulation 1962; 26: 7-11.
5 Dubois E L, Tuffanelli D L. Clinical manifestations of systemic lupus erythematosus: computer analysis of 520 systemic lupus erythematosus: co
cases. $F A M A$ 1964; 190: 104-11.

6 Klinkhoff A V, Thompson C R, Reid G D, Tomlinson C W. $M$-mode and two-dimensional echocardiographic abnormalities in systemic lupus erythematosus. JAMA 1985 253: 3273-7.

7 Doherty N E, Feldman G, Maurer G, Siegel R J. Echocardiographic findings in systemic lupus erythematosus. Am 7 Cardiol 1988; 61: 1144.

8 Galve E, Candell-Riera J, Pigrau C, et al. Prevalence, morphologic types, and evolution of cardiac valvular disease in systemic lupus erythematosus. $N$ Engl $\mathcal{f}$ Med 1988; 319: 817-23.

9 Maniscalco B S, Felner J M, McCans J L, Chiapella J A. Echocardiographic abnormalities in systemic lupus erythematosus [abstract]. Circulation 1975; 52 (suppl II): erythematc
10 Elkayam U, Weiss S, Lainado S. Pericardial effusion and mitral valve involvement in systemic lupus erythematosus: echocardiographic study. Ann Rheum Dis 1977; 36: 349-53.

11 Asherson R A, Gibson D G, Evans D W, Baguley E, Hughes G R V. Diagnostic and therapeutic problems in two patients with antiphospholipid antibodies, heart valve patients with antiphospholipid antibodies, heart valve
lesions, and transient ischaemic attacks. Ann Rheum Dis lesions, and transient

12 Gil A, Khamashta M A, Oliver J, et al. Doppler and twodimensional echocardiographic findings in systemic lupus erythematosus: antiphospholipid antibodies associated with endocardial disease and mitral insufficiency [abstract]. Clin Exp Rheumatol 1988; 6: 204

13 Ford P M, Ford S E, Lillicrap D P. Association of lupus anticoagulant with severe valvular heart disease in systemic lupus erythematosus. $\mathcal{F}$ Rheumatol 1988; 15: 597-600.

14 Tan E M, Cohen A S, Fries J, et al. The 1982 revised criteria for classification of SLE. Arthritis Rheum 1982; 25: 1271-7.

15 American Rheumatism Association Glossary Committee. Signs and symptoms. Dictionary of the Rheumatic Diseases 1982; 1: 1-80.

16 Feigenbaum H. Echocardiography. 4th ed. Philadelphia: Lea and Febiger, 1986: 127-87.

17 Loizou S, McCrea J D, Rudge A C, et al. Measurement of anticardiolipin antibodies by an enzyme-linked immunosorbent assay (ELISA): standardization and quantitation of results. Clin Exp Immunol 1985; 62: 728-45.

18 Gharavi A E, Harris E N, Asherson R A, Hughes G R V. Anticardiolipin antibodies: isotype distribution and phospholipid specificity. Ann Rheum Dis 1987; 46: 1-6.

19 Cervera R, Font J, López-Soto A, et al. Isotype distribution of anticardiolipin antibodies in systemic lupus erythema tosus: prospective analysis of a series of 100 patients. Ann Rheum Dis 1990; 49: 109-13.

20 Cowchock S, Fort J, Muñoz S, Norberg R, Maddrey W. False positive ELISA tests for anticardiolipin antibodies in sera from patients with repeated abortion, rheumatologic sera from patients with repeated abortion, rheumatologic disorders and primary biliary cirrhosis: correlation with elevated polyclonal IgM and implications for patients with
repeated abortion. Clin Exp Immunol 1988; 73: 289-94.

21 Exner T, Rickard K A, Kronenberg H. Studies on phos pholipids in the action of a lupus coagulation inhibitor. Pathology 1975; 7: 319-28.

22 Libman E, Sacks B. A hitherto undescribed form of valvula and mural endocarditis. Arch Intern Med 1924; 33: 701-37.

23 Mandell B F. Cardiovascular involvement in systemic lupus erythematosus. Semin Arthritis Rheum 1987; 17: 126-41.

24 Straaton K V, Chatham W W, Reveille J D, Kooping W J, Smith S H. Clinically significant valvular heart disease in
systemic lupus erythematosus. $A m \mathcal{F} M e d$ 1988; 85: 645-50.

25 Moynihan T, Hansen R, Troup P, Olinger G. Simultaneous aortic and mitral valve replacement for lupus endocarditis: aortic and mitral valve replacement for lupus endocarditis: report of a case and review of the
Cardiovasc Surg 1988; 95: 142-5.

26 Anderson $\mathrm{D}$, Bell $\mathrm{D}$, Lodge $\mathrm{R}$, et al. Recurrent cerebra ischemia and mitral valve vegetation in a patient with lupus anticoagulant. $\mathcal{F}$ Rheumatol 1987; 14: 839-41.

27 Lehman T J, Palmeri S T, Hastings C, Klippel J H, Plot P H. Bacterial endocarditis complicating systemic lupus erythematosus. I Rheumatol 1983; 10: 655-8.

28 Hughes G R V. Thrombosis, abortion, cerebral disease and upus anticoagulant. $B M 7$ 1983; 287: 1088-9.

29 Harris E N, Gharavi A R, Boey M L, et al. Anticardiolipin antibodies: detection by radioimmunoassay and association with thrombosis in systemic lupus erythematosus. Lancet 1983; ii: $1211-4$.

30 Lockshin M D, Druzin M L, Goei S, et al. Antibody to cardiolipin as a predictor of fetal distress or death in pregnant patients with systemic lupus erythematosus. $N$ pregnant patients with systemic

31 Khamashta M A, Harris E N, Gharavi A E, et al. Immune mediated mechanism for thrombosis: antiphospholipid antibody binding to platelet membranes. Ann Rheum Dis 1988; 47: 849-54.

32 Carreras L O, Defreyn G, Machin S J, et al. Arteria thrombosis, intrauterine death and lupus anticoagulant: detection of immunoglobulin interfering with prostacyclin formation. Lancet 1983; i: 244-6.

33 Shapiro R F, Gamble C N, Wiesner K B, et al. Immunopathogenesis of Libman-Sacks endocarditis. Assessment by light and immunofluorescent microscopy in two patients. Ann Rheum Dis 1977; 36: 508-16. 